\title{
Renal calyceal diverticulum mimicking a large renal cyst-A case report
}

\section{Samson Ravirajendran ${ }^{1}$, Neelakandan Ramasamy ${ }^{2}$, Chandru Thirunavukkarasu ${ }^{3}$, Natarajan Kumaresan ${ }^{4}$}

${ }^{1}$ Senior Registrar, ${ }^{2}$ Assistant Professor, ${ }^{3}$ Professor, ${ }^{4}$ Professor and Head, Department of Urology, Sri Ramachandra Institute of Higher Education and Research (SRIHER), Porur, Chennai 600116, Tamil Nadu, India

(Received: May $2020 \quad$ Revised: June $2020 \quad$ Accepted: July 2020)

Corresponding author: Neelakandan Ramasamy. Email: dr.neelakandan@gmail.com

\begin{abstract}
Renal calyceal diverticulum are outpouching from renal calyx or pelvis into the renal cortex lined by transitional cell epithelium. We report a case of 35 years old male with history of accidental fall from bike, who presented with persistent right loin pain and hematuria. CT Urogram showed multiple large right renal cysts (BOSNIAK II). Patient was planned laparoscopic renal cyst deroofing. Due to suspicion of calyceal diverticulum, an intraoperative Retrograde Pyelogram (RGP) was done which showed calyceal diverticulum. Laparoscopic calyceal diverticulotomy with closure of communication was done. If only a cyst deroofing was done considering the CT finding alone, it would have ended up in post-operative complication of urinoma which may require a re-surgery. This case report emphasizes the importance of anticipating calyceal diverticulum and intraoperative attempts in the form of doing RGP to identify calyceal diverticulum which mimic renal cyst regardless of CT finding in suspected cases.
\end{abstract}

Keywords: Calyceal diverticulum; large renal cyst; retrograde pyelogram; laparoscopic calyceal; Diverticulotomy.

\section{INTRODUCTION}

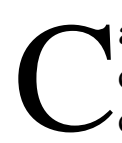

alyceal diverticulum are urine containing cavities in the renal parenchyma which communicate with the pelvicalyceal system by an infundibulum. They are benign, usually asymptomatic and often found incidentally on radiological imaging. Calyceal diverticulum can be diagnosed with delayed films in contrast CT by filling of contrast into the diverticulum. If the communicating neck is not patent, there will not be any reflux of contrast during CT imaging and it poses a diagnostic challenge in radiological diagnosis. Here we present a similar case in which the incidental finding of a large renal cyst later turned out to be a calyceal diverticulum.
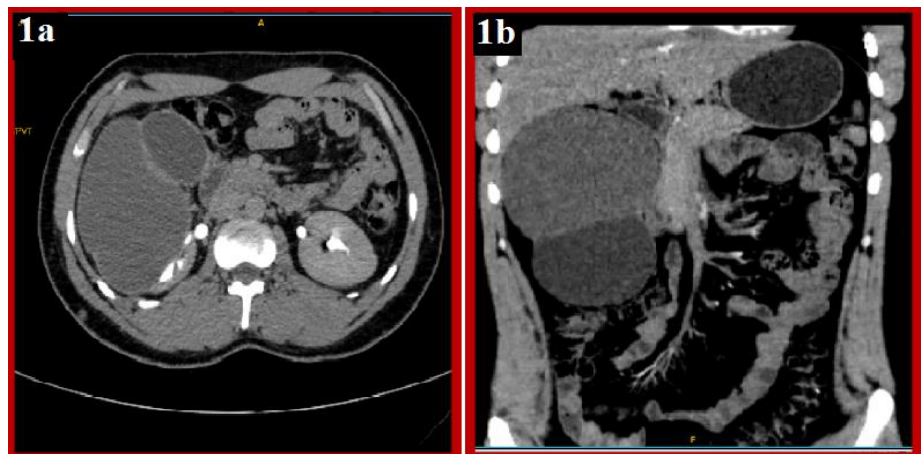

Fig. 1a \& 1b: Axial and coronal section of contrast CT showing large right renal cysts with minimal enhancement of diverticulum.
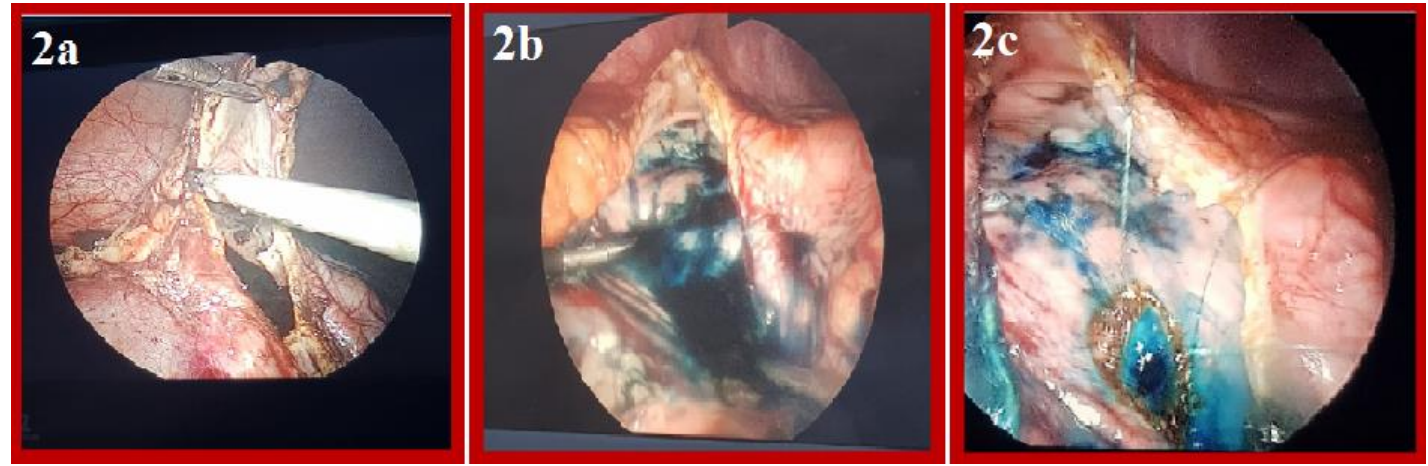

Fig. 2: Intra operative picture during laparoscopy showing (a) Hemorrhagic fluid inside the diverticulum after excision of the wall. (b) Methylene blue dye extravasation into the diverticulum. (c) Laparoscopic closure of calyceal diverticular neck.

\section{CASE REPORT}

A thirty-five years old male presented to outpatient department with dull aching localized right loin pain for two weeks following a fall from bike. He had two episodes of total painless hematuria. On examination patient was comfortable, no pallor, pulse rate - 
86/min, blood pressure - 110/70mmHg, Abdominal examination revealed a vague mass in the right lumbar region with minimal right renal angle tenderness. Random blood sugar $-108 \mathrm{mgs} / \mathrm{dl}$, Hb$10 \mathrm{~g} \%$, Blood Urea Nitrogen - 16mgs/dl, Serum creatinine $-0.8 \mathrm{mgs} / \mathrm{dl}$. Urine routine showed plenty of Red blood cells. CT Urogram reported three large right renal cysts of size $12 \times 7 \mathrm{~cm}, 6 \times 5 \mathrm{~cm}$ and $4 \times 3 \mathrm{~cm}$ (Fig.1). Patient was initially managed conservatively. In view of persistent pain, patient was planned for laparoscopic deroofing of the cyst. As the cysts were unilateral, large and unusual for the age and closer to collecting system, the possibility of a calyceal diverticulum was suspected. So, an intraoperative RGP was done, which revealed communication of cyst with the collecting system. Laparoscopic calyceal diverticulotomy was done, about $400 \mathrm{cc}$ of hemorrhagic fluid aspirated, communication confirmed by injecting methylene blue through retrograde ureteric catheter from below. Closure of communication done (Fig. 2). Double J uretric stent was placed. Patient recovered well. Per urethral Foley's catheter removed on $3^{\text {rd }}$ postoperative day. Abdominal drain removed on $10^{\text {th }}$ postoperative day. Double J stent was removed after 3 weeks. Patient was doing well on postoperative follow up.

\section{DISCUSSION}

Calyceal diverticulum was first described by Rayer in 1841 (1). They are lined by non-secretory transitional epithelium. There is retrograde filling of urine into these cavities from the urinary collecting system. The majority of calyceal diverticulum are asymptomatic and the diagnosis is usually made on imaging done for other reasons. The incidence is around 0.2-0.6\% of the patients undergoing intravenous urogram (2). Meta-analysis studies show that these diverticula are associated with upper pole in $48.9 \%$ versus $29.7 \%$ and $21 \%$ in the mid-pole and lower pole respectively. They are common in women (63\%) than men (37\%) (3). There is no predilection to particular side of the body. They are of varying sizes. Stones are identified in $9.5 \%$ to $50 \%$ of cases (4).

The exact cause of this calyceal diverticulum is unknown. Majority of the investigators favor congenital over an acquired cause. There are two proposed theories supporting the congenital cause, one is failure of one of ureteral branching to stimulate the metanephros resulting in diverticulum $(5,6)$. The other is disordered ureteral branching resulting in failure of higher order of ureteral bud branching to degenerate (7). The potentially acquired causes of calyceal diverticulum are obstructive, neuromuscular, traumatic, fibrotic. However, examination of surgical specimen has failed to provide pathological features which would support the above mentioned acquired causes.

Complications include secondary infection, pain due to stone formation, compression effect on surrounding structures (in cases of large calyceal diverticulum). There is no history and physical examination or laboratory findings that is specific for the diagnosis of calyceal diverticulum. Adequate imaging is required for identification.

Differential diagnoses on imaging include simple cyst, hydrocalyx, parapelvic cyst, tubercular cavity, papillary necrosis, and renal tumor. Calyceal diverticulum are classified as type1, where the diverticulum communicates with the minor calyx or infundibulum and type2, where the diverticulum communicates with major calyx or renal pelvis (8).

Calyceal diverticula may not be seen on plain radiography. They have similar echotexture as renal cyst on ultrasound and are difficult to diagnose unless filled with stones. Intravenous urogram can demonstrate calyceal diverticulum but patency of the diverticular neck should also be considered for opacification. On IVU, calyceal diverticula have the appearance of opacified cystic cavities, which communicate with the renal collecting system (9). Delayed phase imaging on CT or imaging in prone position demonstrates infilling of the diverticulum (10) later than the pelvicalyceal system, since there is a retrograde nondependent filling of contrast into the diverticulum. Thus, a CT imaging can miss a diverticulum (11) as in our case.

Factors arousing a suspicion of diverticulum in our case are

1. Unilateral large cyst, which is unusual for this age.

2. History of hematuria indicate cyst may communicate with collecting system.

3. Cystic lesion closer to pelvicalyceal system.

4. Retrospective review of contrast CT showed a minimal increase in Hounsfield unit in the diverticulum.

Hence, a broad suspicion of calyceal diverticulum should be considered particularly in a large renal cyst with hematuria. Also when a laparoscopic surgery is considered for a large renal cyst or cyst closer to the pelvicalyceal system in the form of cyst deroofing, a differential diagnosis of calyceal diverticulum should always be kept in mind and an intra operative Retrograde Pyelogram prior to laparoscopy and a methylene blue dye injection through the retrograde catheter after opening up the cyst helps in identification of the communication (calyceal diverticulum) even if other preoperative imaging modalities fails to figure out.

\section{CONCLUSION}

Calyceal diverticulum is one of the differential diagnosis of renal cyst. Contrast CT may sometime fail to demonstrate this specific finding. A proper history and a review of CT may help to bring out this condition. Also, the importance of intraoperative 
Retrograde catheterization/ pyelogram during laparoscopic management of renal cyst to identify calyceal diverticulum has been stressed and thereby to close the communication with the urinary system to avoid post-operative complications like urinoma.

\section{CONFLICT OF INTEREST}

Authors declare no conflicts of interest.

\section{REFERENCES}

1. Shapiro, E., Bauer, S. B., Chow, J. S. Anomalies of the upper urinary tract. In: Campbell-Walsh, editor. Urology. 10th ed. Philadelphia: Elsevier-Saunders. 2012; 3153-3154.

2. Mandeville, J. A., Gnessin, E., Lingeman, J. E. Percutaneous management of calyceal diverticula: An American experience. In: Al-Khandari AM, editor. Diffi cult Cases in Endourology. London: Spinger-Verlag. 2013; 33

3. Waingankar, N., Hayek, S., Smith, A. D., Okeke, Z. Calyceal diverticula: a comprehensive review. Rev Urol. 2014; 16(1): 29e43.

4. Timmons, J. W. Jr., Malek, R. S., Hattery, R. R., Deweerd, J. H. Caliceal diverticulum. J Urol. 1975; 114: 6-9.

5. Wulfsohn, M. A. Pyelocaliceal diverticula. J Urol. 1980; 123: $1-8$

6. Middleton, A. W. Jr., Pfister, R. C. Stone-containing pyelocaliceal diverticulum: embryogenic, anatomic, radiologic and clinical characteristics. J Urol. 1974; 111: 26.

7. Narath, P. A. Renal Pelvis and Ureter. New York: Grune and Stratton; 1951.

8. Wulfsohn, M. A. Pyelocaliceal diverticula. J Urol. 1980; 123: $1-8$.

9. Davidson, A. J., Hartman, D. S., Choyke, P. L., Wagner, B. J. Davidson's Radiology of the Kidney and Genitourinary Tract. 3rd ed. Philadelphia: W.B. Saunders Company. 1999; 416-421.

10. Dunnick, N. R., Sandler, C. M., Newhouse, J. H., Amis, E. S. Jr., Textbook of Uroradiology. 4th ed. Philadelphia: Lippincott Williams \& Wilkins. 2008.

11. Mullett, R., Belfield, J. C., Vinjamuri, S. Calyceal diverticulum-a mimic of different pathologies on multiple imaging modalities. J Radiol Case Rep. 2012; 6: 10-17. 